\title{
New taxa and nomenclatorial actions in volume 150
}

Acari

\section{Carabodidae}

Austrocarabodes foliaceisetus georgiensis Murvanidze \& Weigmann, 2007 . . . . . . . . . . . . . . . . . 193

Carabodes djaparidzae Murvanidze \& Weigmann, 2007

\section{Coleoptera}

\section{Carabidae}

Dolichoctis (Spinidolichoctis) nigricauda Baehr, 2007 . 81 Dolichoctis (Spinidolichoctis) picescens Baehr, 2007 . . . 80 Dolichoctis (Spinidolichoctis) sinuaticollis Baehr, 2007 . 79 Paradolichoctis cordicollis vixspinosa Baehr, 2007 . . . . 84

\section{Cicindelidae}

Neochila apicenitens Cassola, 2007 . . . . . . . . . . . 418

Neochila hassoni Cassola, 2007 . . . . . . . . . . . . . 413

Neochila horii Wiesner, 1988 raised to full species . . 408

Neochila kigonserana lindemannae Mandl, syn. n. of $N$.

kigonserana W. Horn ................ 407

Neochila kigonserana viridissima Mandl, syn. n. of N. congoana Mandl ....................... 409

Neochila nigromarginilabris Mandl, syn. $\mathbf{n}$. of $N$. congoana Mandl ... . . . . . . . . . . . . . . . . . . . 409

Neochila nitida Cassola, 2007 . . . . . . . . . . . . . 416

\section{Scarabaeidae}

Amphistomus alfurorum Huijbregts \& Krikken, 2007 . . . .

Ochicanthon crockermontis Krikken \& Huijbregts, 2007.

436

Ochicanthon crypticus Krikken \& Huijbregts, 2007 . . 438

Ochicanthon danum Krikken \& Huijbregts, 2007 . . 458

Ochicanthon dulitmontis Krikken \& Huijbregts, 2007

Ochicanthon dytiscoides (Boucomont, 1914) Lectotype selected

Ochicanthon edmondsi Krikken \& Huijbregts, 2007 . 428

Ochicanthon hanskii Krikken \& Huijbregts, 2007 .. 463

Ochicanthon javanus Krikken \& Huijbregts, 2007 .. 459

Ochicanthon kimanis Krikken \& Huijbregts, 2007. . 456

Ochicanthon mulu Krikken \& Huijbregts, 2007 . . . 455

Ochicanthon neglectus Krikken \& Huijbregts, 2007 . . 443

Ochicanthon peninsularis Krikken \& Huijbregts, 2007 . . .

......................... 439

Ochicanthon rombauti Krikken \& Huijbregts, 2007
Ochicanthon simboroni Ochi et al., 2006 syn. n. of O. cambeforti (Ochi, Kon \& Kikuta, 1997) . 448 Ochicanthon tambunan Krikken \& Huijbregts, 2007 . . . .

\section{Diptera}

\section{Dolichopodidae}

Pharcoura Bickel, 2007 ................... 6

Pharcoura biobio Bickel, 2007 ... . . . . . . . . . . 11

Pharcoura nahuelbuta Bickel, 2007 ............ 8

Pharcoura newthayorum Bickel, 2007 .......... 8

\section{Lauxaniidae}

Sapromyza laszlopappi Merz, 2007 . . . . . . . . . . . 67

Sapromyza undulata Merz, 2007 . . . . . . . . . . . . . . 69

\section{Platystomatidae}

Euprosopia danielsi McAlpine, 2007 . . . . . . . . . . . 228

Euprosopia holmesi McAlpine, 2007 . . . . . . . . . . . 231

Euprosopia tomareae McAlpine, 2007 . . . . . . . . 226

\section{Hemiptera}

\section{Aleyrodidae}

Udamoselis estrellamarinae Martin, $2007 \ldots \ldots \ldots \ldots 15$

\section{Cicadidae}

Purana karimunjawa Duffels \& Schouten, 2007 . . . 383

Purana latifascia Duffels \& Schouten, 2007 . . . . . . 381

Purana metallica Duffels \& Schouten, 2007 . . . . . 373

Purana mulu Duffels \& Schouten, 2007 . . . . . . . . 380

Purana usnani Duffels \& Schouten, 2007 ... . . . . 375

\section{Miridae}

Arbolygus Kerzhner, 1979 syn. n. of Philostephanus Distant, $1909 \ldots \ldots \ldots \ldots$. . . . . . . . . . . 106

Liocapsus brevirostris Poppius, Lectotype selected . . . 166 Liocapsus gotohi Yasunaga \& Schwartz, 2007 . . . . . . 168 Liocapsus langtang Yasunaga \& Schwartz, 2007 . . . . 170 Liocapsus ochromelas Yasunaga \& Schwartz, 2007 . . 172 Mahania Poppius, 1915 gen. rev. . . . . . . . . 172 Mahania elongata Poppius, 1915 comb. rev., Lectotype

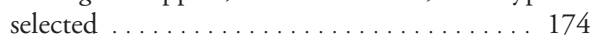
Orientocapsus Yasunaga \& Schwartz, 2007 . . . . . . 156 Orientocapsus aquilus Yasunaga \& Schwartz, 2007 . 157 Orientocapsus bicoloratus Yasunaga \& Schwartz, 2007. 
Orientocapsus cheroti Yasunaga \& Schwartz, $2007 \ldots 161$

Orientocapsus picinus (Lu \& Zheng, 1998) comb. n. . . . . .

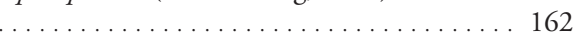

Orientocapsus zhangi (Lu \& Zheng, 1998) comb. n. . . . .

Philostephanus ailaoensis (Lu \& Zheng, 1998, 1998) comb. $n$. 163

Philostephanus castaneus Yasunaga \& Schwartz, 2007 111

. . . . . . . . . . . . . . . . . . . . . . 113

Philostephanus difficilis (Lu \& Zheng, 1998) comb. n.. . .

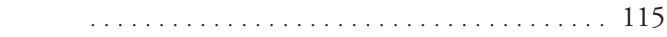

Philostephanus fulvus (Jakovlev, 1882) comb. n. . . . . 117

Philostephanus glaber (Kerzhner, 1988) comb. n. . . . 119

Philostephanus himalayicus (Lu \& Zheng, 1998) comb. n.

Philostephanus longustus (Lu \& Zheng, 1998) comb. n.. .

............................ 124

Philostephanus lucidus Yasunaga \& Schwartz, 2007 . 127

Philostephanus monticola Yasunaga \& Schwartz, 2007 . . .

. . . . . . . ..................... 128

Philostephanus nepalensis Yasunaga \& Schwartz, 2007 . . . .

......................... 131

Philostephanus ovalis Yasunaga \& Schwartz, 2007 . . 131

Philostephanus pilosus Yasunaga \& Schwartz, 2007 . . 133

Philostephanus poppii Yasunaga \& Schwartz, 2007 .. 135

Philostephanus pronotalis (Zheng \& Liu, 1992) comb. n.

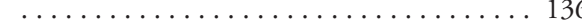

Philostephanus renae (Lu \& Zheng, 1998) comb. n. . . . . .

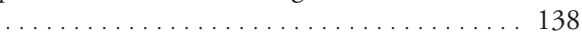

Philostephanus rubripes (Jakovlev, 1876) comb. n. . . 139

Philostephanus sakuraii Yasunaga \& Schwartz, 2007

Philostephanus taiwanensis Yasunaga \& Schwartz, 2007.

Philostephanus tibialis (Lu \& Zheng, 1998) comb. n. . . .

........................... 146

Philostephanus ulmi (Kerzhner, 1978) comb. n. . . . . 147

Philostephanus variegatus (Poppius, 1914) comb. n., Lec-

totype selected .................. . 149

Philostephanus vitaliter Distant, 1909, Lectotype selected 151

Philostephanus wuzhiensis (Lu \& Zheng, 1998) comb. n.

\section{Reduviidae}

Chinemesa uniannulata Rédei, 2007.

214

\section{Lepidoptera}

\section{Arctiidae}

Leptosoma absurdum Swinhoe, 1892 syn. n. of Nyctemera clathratum (Snellen van Vollenhoven, 1863) . . 44

Nyctemera dauila De Vos, 2007 . . . . . . . . . . . . 49

Nyctemera giloloensis De Vos, 2007 . . . . . . . . 45

Nyctemera latimargo (Rothschild, 1915), Lectotype selected .................... 49

Nyctemera oninica De Vos, 2007 . . . . . . . . . 46

Nyctemera pratti (Bethune-Baker, 1904) syn. n. of Nyctemera mesolychna Meyrick, 1889, Lectotype selected

\section{Orthoptera}

\section{Tettigoniidae}

Hemielimaea (H.) caricercata Ingrisch, 2007 . . . . . 96 Hemielimaea $(H$.$) chinensis Brunner von Wattenwyl,$ 1878, Lectotype selected ... . . . . . . . . . 89

Hemielimaea (H.) kuatun Ingrisch, 2007 . . . . . . . . 96

Hemielimaea (H.) omeishanica Gorochov, 2007 . . . . 97

Hemielimaea (H.) mannhardti (Krausze, 1903), restored to full species status . . . . . . . . . . . . . 98 Hemielimaea (Pseudelimaea) cucullata Ingrisch, 1990 syn. n. of $H$. (P.) procera Ingrisch, $1990 \ldots \ldots$. . . 98

\section{Thysanoptera}

\section{Phlaeothripidae}

Haplothrips (Gigaplothrips) Priesner, 1949 syn n. of Neoheegeria Schmutz, $1909 \ldots \ldots \ldots$. . . . . . 60

Haplothrips biroi (Priesner, 1928) comb. n. . . . . . 58 Haplothrips faurei (Zur Strassen, 1966) comb. n. . . . 58 Haplothrips hrasvamukha (Ramakrishna, 1928) comb. n. .

Haplothrips johni (Priesner, 1925) comb. . . . . . . 59

Haplothrips lederi (Priesner, 1924) comb. $\mathbf{n}$.

Haplothrips verbasci (Osborn, 1897) comb. n. . . . . . 59

Neoheegeria ballotae Priesner, 1951 syn n. of $N$. dalmatica Schmutz, $1909 \ldots \ldots \ldots \ldots \ldots \ldots \ldots . \ldots 60$

Neoheegeria gigantea (Priesner, 1934) comb. n. . . . . 62

Neoheegeria hamanni Priesner, 1961 syn n. of $N$. dalmatica Schmutz, $1909 \ldots \ldots \ldots \ldots$. . . . . . . . . 60

Neoheegeria nevskyi Moulton, 1946 syn n. of $N$. dalmatica Schmutz, 1909 ................... 60

Xylaplothrips montanus (Ananthakrishnan \& Jagadish, 1970). comb. n. . . . . . . . . . . . . . 59 